\title{
Clinical Exome Sequencing Enables Congenital Sialidosis Type II Diagnosis in Two Siblings Presenting with Unreported Clinical Features from a Rare Homozygous Sequence Variant p.(Tyr370Cys) in NEU1
}

\author{
Elda Ariadna Flores-Contreras ${ }^{a} \quad$ José Elías García-Ortiz ${ }^{b}$ Carla Daniela Robles-Espinozac, d \\ Viviana Zomosa-Signoret ${ }^{a}$ Luis Eduardo Becerra-Solano ${ }^{e}$ Román Vidaltamayo $^{f}$ \\ Carolina Castaneda-Garcíac Eduardo Esparza-Garcíag Christian Molina-Aguilar ${ }^{c}$, $h$ \\ Angélica Alejandra Hernández-Orozco ${ }^{\mathrm{b}}$ Carlos Córdova-Fletes ${ }^{\mathrm{a}}$
}

\begin{abstract}
aDepartamento de Bioquímica y Medicina Molecular, Facultad de Medicina, Universidad Autónoma de Nuevo León, Monterrey, Mexico; 'División de Genética, Centro de Investigación Biomédica de Occidente, CMNO-IMSS, Guadalajara, Mexico; ‘ Laboratorio Internacional de Investigación sobre el Genoma Humano, Universidad Nacional Autónoma de México, Campus Juriquilla, Santiago de Querétaro, Mexico; 'Wellcome Sanger Institute, Hinxton, UK; eDivisión de Ciencias Biomédicas, Departamento de Clínicas, CUALTOS Universidad de Guadalajara, Tepatitlán, Mexico; 'Department of Basic Science, School of Medicine, Universidad de Monterrey, San Pedro GG, Mexico; 9UMAE Hospital de Pediatría, CMNO-IMSS, Guadalajara, Mexico; ${ }^{\mathrm{h} C e n t r e ~ o f ~ B i o e n g i n e e r i n g, ~ S c h o o l ~}$ of Engineering and Sciences, Tecnologico de Monterrey, Monterrey, Mexico
\end{abstract}

\section{Established Facts}

- Sialidosis is a rare autosomal recessive disease that presents progressive lysosomal storage of sialylated glycopeptides and oligosaccharides caused by homozygous or compound heterozygous sequence variants in the neuraminidase 1 (NEU1) gene.

- According to their type and location, these sequence variants can lead to 2 main sialidosis clinical variants: sialidosis type I and II. Sialidosis type II is the most severe and presents at prenatal stage and/or early age.

\section{Novel Insights}

- We report a 4-member pedigree segregating an ultra-rare missense variant (c.1109A>G; p.Tyr370Cys) in NEU1 as detected by whole-exome sequencing.

- Two short-lived siblings presented, among several other clinical features, with corpus callosum hypoplasia, corneal opacity, hernia, dysostosis, and/or ventricular septal hypertrophy previously unreported from such homozygous sequence variant. These patients represent the third and fourth cases, respectively reported so far, but the first case of affected siblings inheriting this sequence variant.

- We also present a novel molecular model exhibiting the consequences of the Y370C substitution in the sialidase-1 tridimensional structure.

Correspondence to:

Carlos Córdova-Fletes, carlos.cordovafl@uanl.edu.mx 


\section{Keywords}

Clinical exome sequencing · Sialidosis diagnosis · Phenotypic variability · Sialidase- 1 - Rare variant . Tridimensional modeling

\begin{abstract}
Sialidosis is a rare autosomal recessive disease that presents with progressive lysosomal storage of sialylated glycopeptides and oligosaccharides caused by homozygous or compound heterozygous sequence variants in the neuraminidase 1 (NEU1) gene. These sequence variants can lead to sialidosis type I and II; the latter is the most severe and presents prenatally or at early age. However, sialidosis diagnosis is challenging, especially in those health systems with limited resources of developing countries. Consequently, it is necessary to dip into high-throughput molecular diagnostic tools to allow for an accurate diagnosis with better cost-effectiveness and turnaround time. We report a 4-member pedigree segregating an ultrarare missense variant, c.1109A > G; p.Tyr370Cys, in NEU1 as detected by whole-exome sequencing. Two short-lived siblings, who presented with previously unreported clinical features from such a homozygous sequence variant, were diagnosed with sialidosis type II. Additionally, we present a novel molecular model exhibiting the consequences of the variant in the sialidase- 1 tridimensional structure. This study allowed us to provide a definitive diagnosis for our patients, increase our understanding of this pathogenic variant, and improve genetic counseling.
\end{abstract}

(c) 2021 S. Karger AG, Basel

\section{Introduction}

Sialidosis (OMIM 256550) is a rare autosomal recessive disease that characterized by progressive lysosomal storage of sialylated glycopeptides and oligosaccharides caused by homozygous or compound heterozygous sequence variants in the NEU1 gene, located at 6p21.33 [Bonten et al., 1996; Pshezhetsky et al., 1997; d'Azzo et al., 2015; Khan and Sergi, 2018]. NEU1 encodes for an a-neuraminidase enzyme (also known as sialidase-1 or lysosomal sialidase) that strictly activates by forming a multiprotein complex with $\beta$-galactosidase (encoded by GLB1) and lysosomal protective protein/cathepsin A (encoded by CTSA) [Franceschetti and Canafoglia, 2016; Annunziata and d'Azzo, 2017; Maroofian et al., 2018]. NEU1 is a negative regulator of lysosomal exocytosis and processes sialic acid from membrane glycoproteins, such as lysosomal membrane protein 1 (LAMP1). LAMP1 is an integral membrane protein that docks to lysosomes at the plasma membrane, allowing for lysosomal exocytosis. Thus, loss of function or deficiency of NEU1 leads to the accumulation of oversialylated LAMP1, resulting in increased LAMP1-marked lysosomes and excessive lysosomal exocytosis [Yogalingam et al., 2008]. Ultimately, patients present aberrant tissue accumulation and abnormal excretion of sialylated oligosaccharides in urine, leading to sialidosis [d'Azzo et al., 2015]. Sialidosis has an incidence of 0.04 in 100,000 individuals [Arora et al., 2020] (www.orpha.net) and, to date, 72 disease-causing sequence variants in NEU1 have been reported in the Human Gene Mutation Database (http://www.hgmd. cf.ac.uk/ac/index.php). According to their type, location, and residual activity, these mutations can lead to 2 sialidosis types (type I and II). Sialidosis type I presents in adulthood (second and third decade of life), exhibiting signs such as progressive visual loss, gait abnormalities, bilateral cherry-red macular spots, ataxia, and myoclonic epilepsy. On the other hand, sialidosis type II occurs at prenatal stage and/or early age (prenatal/congenital, infantile, and juvenile subtypes), and is mainly characterized by the presence of hepatosplenomegaly, coarse face, dysostosis multiplex, cherry-red macular spots, and developmental delay/intellectual disability [d'Azzo et al., 2015; Khan and Sergi, 2018].

Given that several of the clinical features seen in sialidosis (both types) also present in many other lysosomal disorders and the fact that routine laboratory tests in most health systems of developing and/or medium- and low-income countries are limited, it is often necessary to dip into alternative high-throughput molecular diagnostic tools such as whole-exome sequencing (WES) to allow for an accurate diagnosis [Maroofian et al., 2018]. In this study, we report a 4-member pedigree segregating a missense sequence variant in NEU1 as detected by WES; 2 short-lived siblings, who presented with previously unreported clinical features from such homozygous sequence variant, were hence diagnosed with sialidosis type II. These patients represent the third and fourth cases, respectively reported so far, but the first case of affected siblings inheriting this pathogenic variant.

\section{Case Presentations}

Patient 1

An 11-month-old male patient was referred to the Genetics Service due to potential lysosomal disease. His mother, 25 years old (G2, C1, P1, A0), and his father, 30 years old, were apparently healthy and nonconsanguineous, with no significant family clini- 
cal history. He was the first gestation of this couple, with prenatal care since the 3rd week of gestation with uneventful pregnancy until the 20th week when hydrops fetalis was identified by ultrasonography. The pregnancy was interrupted at 35 weeks of gestation by rupture of membranes; the patient was born by cesarean section with a weight of $3,250 \mathrm{~g}$ (90th percentile) and length of $50 \mathrm{~cm}$ (90th percentile); his abdomen diameter was $50 \mathrm{~cm}$. At birth, he had ascites, bilateral hydrocele, and ventricular arrhythmia; a few days after, he developed nosocomial multidrug-resistant pneumonia and aspiration pneumopathy. After clinical studies, hepatosplenomegaly, bone dysostosis in spine, and cardiological abnormalities (i.e., ventricular septal hypertrophy and aortic dilation) were confirmed.

At physical examination, he exhibited weight, height, and head circumference of $9.5 \mathrm{~kg}(Z-1.3), 72 \mathrm{~cm}(Z-3.1)$, and $45.6 \mathrm{~cm}(Z$ -2.3 ), respectively, as well as developmental delay, slight flattening of the occiput, high implantation of the scalp, coarse face, facial hypertrichosis and synophrys, broad forehead, horizontal palpebral fissures, corneal opacity, depressed nasal bridge, long philtrum, microretrognathia with relative macroglossia (without protrusion), a high palate, borderline implantation of the ears, short neck, bell-shaped thorax, left infraclavicular capillary malformation, bulging abdomen without collateral vascularity, a midline secondary incisional hernia, hepatomegaly and splenomegaly. $\mathrm{He}$ also presented with male genitals with non-tension bilateral hydrocele, column with dorsal hump and wide sacral fossa, generalized hypotonia, and livedo reticularis. Other findings were corpus callosum hypoplasia and acute renal failure. He died at 2 years old.

\section{Patient 2}

The index case's brother was born at 34 weeks of gestation. He presented with severe ascites, bilateral inguinal hernia, cerebral atrophy, bronchomalacia and cholestatic syndrome; he died at 3 months and could not directly be evaluated by the Genetics Service.

\section{Methods and Results}

Due to hydrops fetalis in the index case, a karyotype from amniotic liquid cells was performed, which was normal (46,XY). Similarly, available lysosomal studies for Gaucher disease, lysosomal acid lipase deficiency, and mucopolysaccharidoses I, II, VI, and VII were all negative. Therefore, to ascertain pathogenic variants related to his clinical phenotype, WES was carried out using the TruSeq Rapid Exome Library Prep kit according to the manufacturer's instructions (Illumina, San Diego, CA, USA). This kit contains $>340,000$ probes of 95 mer covering a $45-\mathrm{Mb}$ region of 214,405 exons (coding exons and neighboring intronic sequences). The WES analysis showed the homozygous missense variant c. $1109 \mathrm{~A}>\mathrm{G}$ (total read depth = 164; GQX quality score $=99$ ) in exon 6 of NEU1 (Fig. 1a) (https://gnomad.broadinstitute.org/ variant/6-31827635-T-C?dataset=gnomad_r2_1), resulting in the amino acid change p.(Tyr370Cys) (Y370C, Fig. 1b; 2a-d). This variant was in silico predicted to be deleterious, probably damaging, and disease causing by SIFT (https://sift.bii.a-star.edu.sg/), Polyphen-2 (http://genetics.bwh.harvard.edu/pph2/), CADD (https://cadd.gs.washington.edu/), and Mutation taster programs (http://www.mutationtaster.org/), respectively. Furthermore, the wild type nucleotide exhibited a high grade of conservation as pro- vided by phyloP/phastCons scores (4.568/1). This variant is annotated in dbSNP as rs1310267862, and has a heterozygous Latino frequency of $1 / 34,480(0.00002900)$, but a global frequency of $1 / 246566(0.000004056)$ according to the gnomAD browser where no homozygous cases have been reported. In addition, as this pathogenic variant had not yet been reported in the ClinVar database (http://www.ncbi.nlm.nih.gov/clinvar/), we have now submitted it there (submission ID: SCV001443261.1). Sanger sequencing electropherograms (using the pair of primers F-NEU1 $5^{\prime}$ GATACACTAAGGCCCCGTGA $3^{\prime}$ and R-NEU1 $5^{\prime}$ CCTGAAGGCAGAGTCCTGAA $3^{\prime}$ ) confirmed the results obtained by WES in the index case and showed that his brother also had the same homozygous missense variant as well that their healthy parents were heterozygous for the pathogenic variant (Fig. 1b). No other likely pathogenic or pathogenic variants were found in NEU1 or other related enzyme genes such as CTSA, GLB1, IDUA, LIPA, $I D S, G B A, A R S B$, or GUSB of the proband. Additionally, as no other homozygous pathogenic variants appeared in the filtering, we discarded major runs of homozygosity modifying the phenotype.

As previously predicted, the $\mathrm{Y} 370 \mathrm{C}$ variant alters the tridimensional structure of the active site of the enzyme, eliminating the benzyl ring and hydroxyl group of this well-preserved amino acid residue. This tyrosine residue stabilizes the transition of a carbonium ion intermediate during catalysis. The crystal structure of a bacterial sialidase (from Salmonella typhimurium LT2) shows the same fold as an influenza virus neuraminidase [Crennell et al., 1993], and its loss leads to an unactive enzyme that still localizes to the lysosome [Bonten et al., 2000]. In our in silico analysis (Fig. 2ad), which was generated based on the crystal structure of the Streptococcus pneumoniae neuraminidase (access no. 2 w20) and where both the energy minimization and structure fitting were achieved using the Swiss-model software suite [Guex et al., 2009; Artimo et al., 2012], we show that the Y370C substitution introduces a side chain incapable of reaching into the active site pocket formed by well-conserved arginine (R76, R278 and R339) and glutamate (E392 and E262) residues, thus leading to the inability of the cysteine residue to stabilize the transition state of the substrate. This leads to a suppression of the catalytic activity of the Y370C mutant product [Bonten et al., 2000].

\section{Discussion and Conclusion}

Establishing a diagnosis for the patients discussed here represented a challenge. It was mainly due to the fact that some clinical features present in these patients overlap with those seen in either other lysosomal diseases (e.g., mucopolysaccaridoses) or mucolipidoses. Additionally, the biochemical tests in our health system are limited, and we have access to only a few of them in a unique reference center in our country. In this regard, most of these tests are focused on the most frequent lysosomal diseases, leaving several other patients undiagnosed, who therefore lose the chance to access a definitive (or palliative) treatment (as for our patients). Hence, we believe that alterna- 


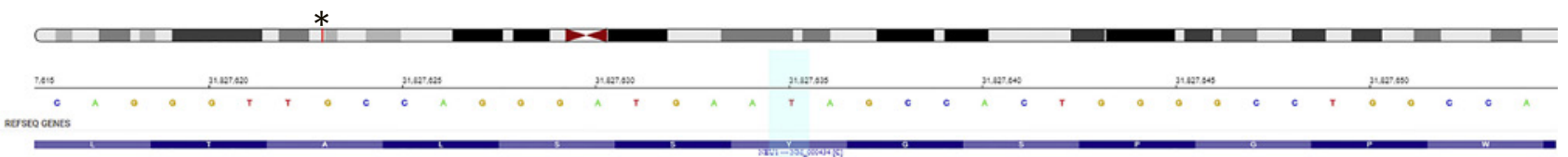

teourtosi necos

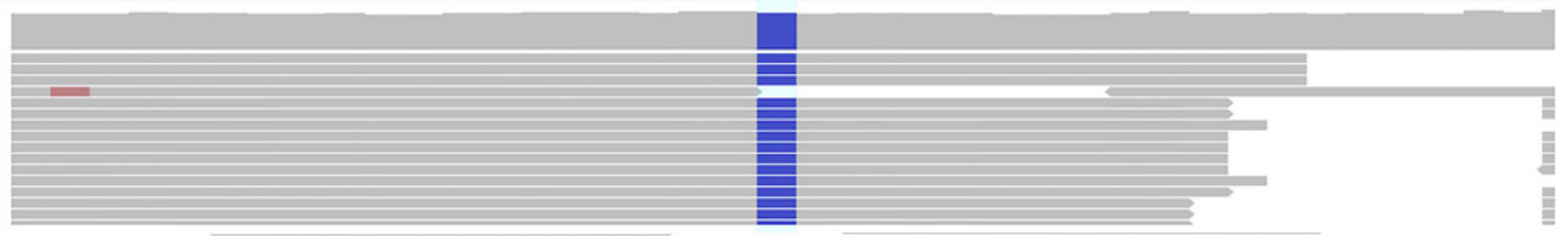

a

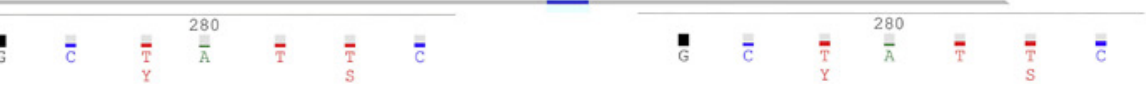

I
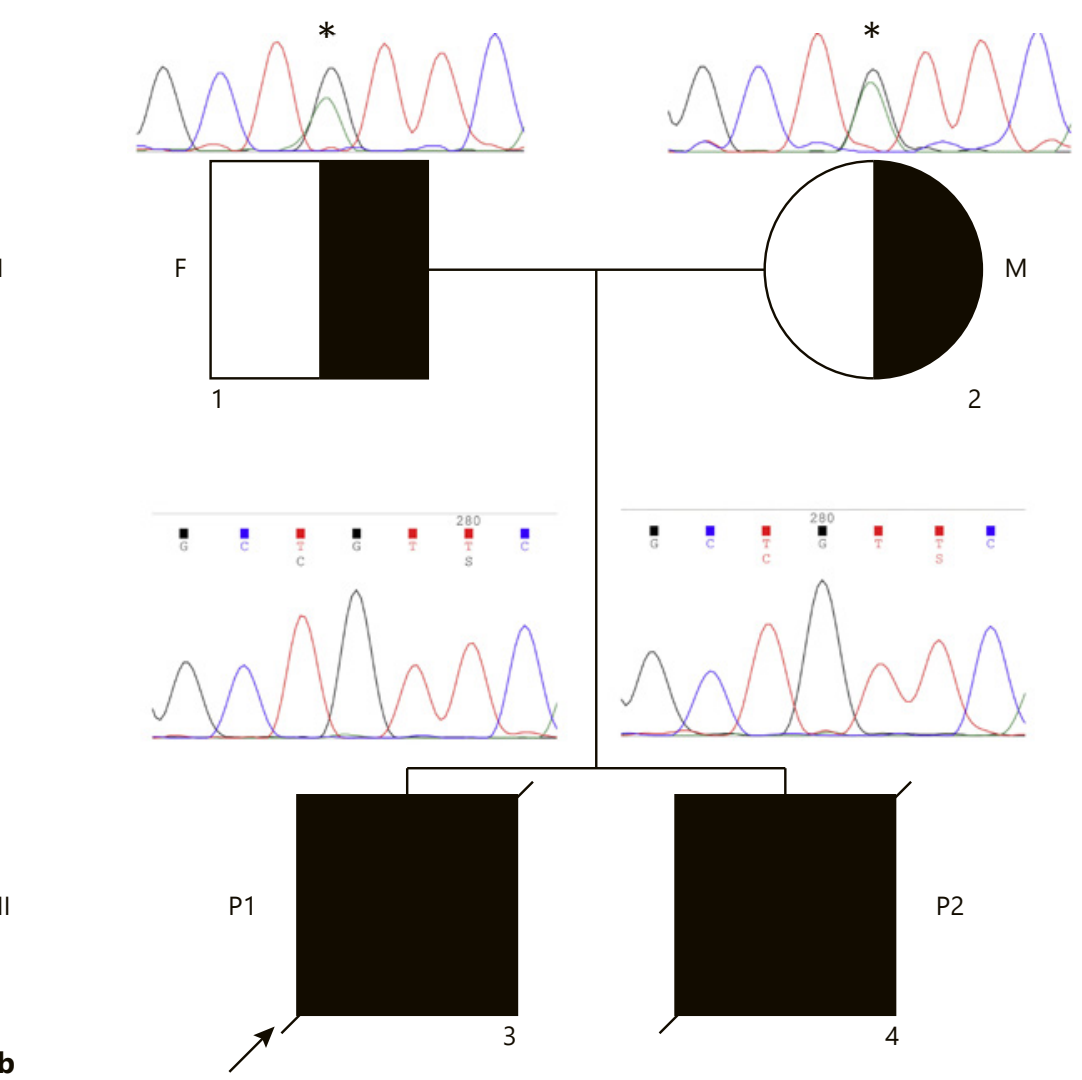

b

Fig. 1. Identification and validation of the NEU1 variant. a Partial screenshot showing the visualization of the c. $1109 \mathrm{~A}>\mathrm{G}$ ( $\mathrm{T}>\mathrm{C}$ in the complementary) variant from VI platform (Illumina). b Family pedigree showing carrier (I:1 and I:2) and affected individuals (II:3 and II:4) and their respective chromatograms from Sanger sequencing. The index case (II:3) is marked by an arrow. * Denotes the pathogenic variant position. F, father; M, mother; $\mathrm{P} 1$, patient 1 (index case); P2, patient 2. 


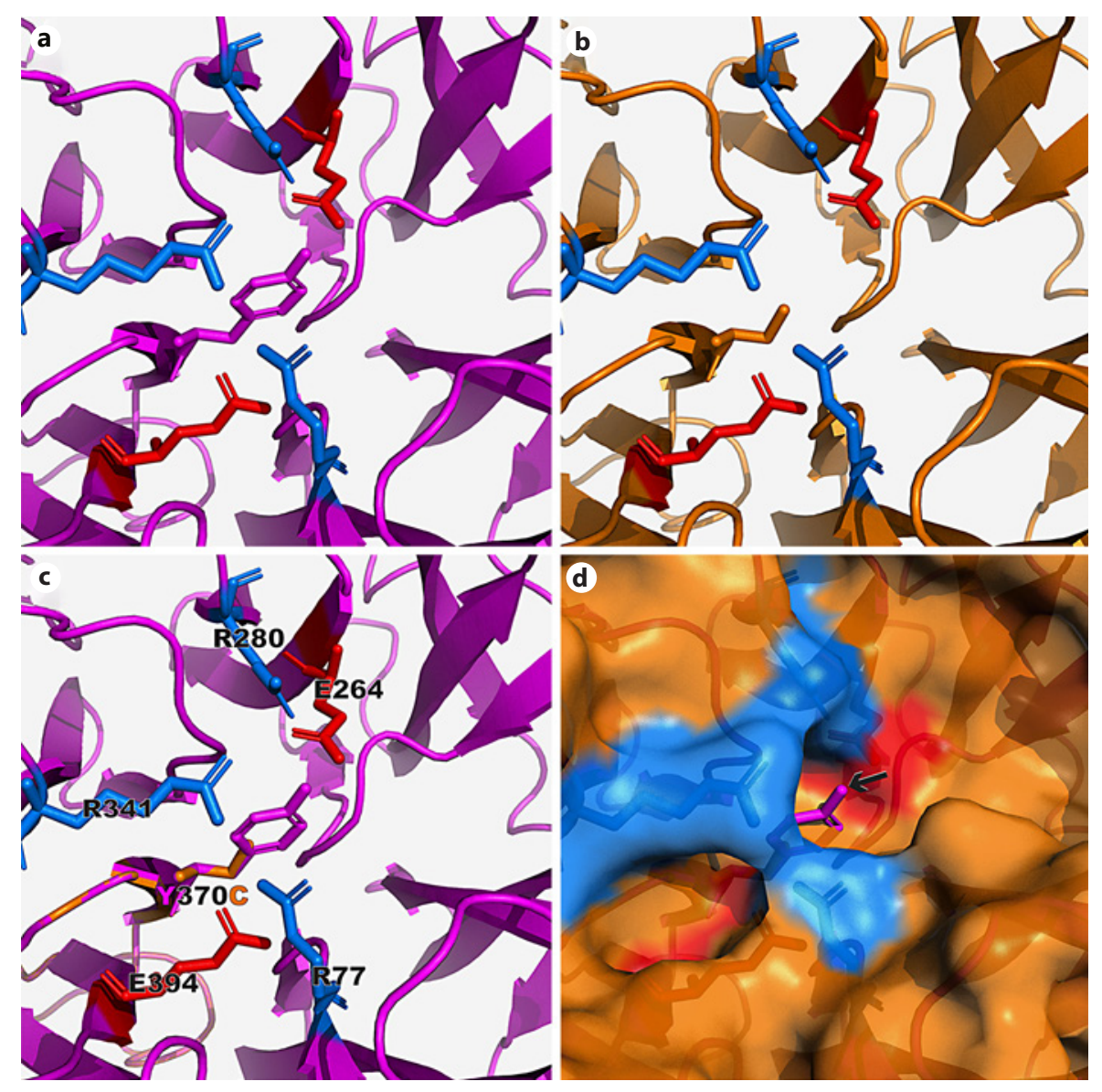

Fig. 2. The Y370 sequence variant in NEU1 does not alter the overall secondary structure of the area surrounding the substrate binding pocket of the enzyme but decreases the height of the floor of the pocket necessary for catalytic activity. Comparison of the tridimensional structures predicted for the wild-type (Y370) NEU1 product (a) and the mutant (C370) likely expressed by the patients (b). Superposition of both structures shows little to no modification of the $\beta$ sheet containing residue 370 (magenta $\beta$ sheet arrowheads in $\mathbf{c}$ ), but the bulkier tyrosine residue ( $\mathrm{Y}$ and magenta in $\mathbf{c}$ ) reaches higher into the pocket formed by 3 well-conserved arginine residues (R77, R280, and R341 in c) that stabilize the carbox- yl group of the neuraminic acid substrate, and that in turn are kept in place by 2 well-conserved glutamate residues (E264 and E394 in c). Representation of the surface occupied by the polypeptide chain demonstrates that C370 forms a shallower floor of the substrate binding pocket (orange surface in $\mathbf{d}$ ) and that $\mathrm{Y} 370$ reaches roughly $40 \%$ higher into the pocket (arrow in d), better serving for the stabilization of the transient intermediate product of catalysis. The visualization (including the renders superposing the mutant over the wild-type structure) and the introduction of the Y370C point variant were using the PyMOL molecular visualization system (OS $\mathrm{X}$ v. 2.4, Schrödinger; San Diego, CA, USA). tive diagnostic tools such as next generation sequencing technologies can improve diagnosis in our country, particularly given its cost-effectiveness and quick turnaround time.

The homozygous variant c.1109A $>\mathrm{G}$ in exon 6 of NEU1 found here is extremely rare, as it has been reported in the gnom $A D$ collection only once in one heterozygous individual of Latino ethnicity. Even though the parents denied consanguinity, this fact suggests that they would share a recent common ancestor, probably with founder effect; however, according to the available data, as well as a ho- mozygosity analysis in the proband, we hypothesize that the relationship between them is not so close. On the other hand, this variant was originally described by Bonten et al. [2000] (but reported as c.1107A>G) in one homozygous patient diagnosed with sialidosis type II and it was related to a severe form of the disease, starting at the fetal stage and associated to infant mortality. Bonten et al. [2000] determined, according to structural and functional analyses, that Tyr370 is one of the active site residues of lysosomal neuraminidase. Twenty years later, just another patient with the same homozygous pathogenic variant has 
Table 1. Comparison of clinical features among patients with the p.(Tyr370Cys) sequence variant reported so far

\begin{tabular}{|c|c|c|c|}
\hline Reference & Bonten et al. [2000] & Maroofian et al. [2018] & This report \\
\hline Cases reported with p.(Tyr370Cys) & 1 & 1 & 2 (siblings) \\
\hline Gender & Male & Female & Male (both patients) \\
\hline Origin & Hispanic-American & Iranian & Hispanic-American \\
\hline Parental consanguinity & Not reported & Yes (first-cousin parents) & Apparently nonconsanguineous \\
\hline Clinical type & Type II infantile & Type II infantile & Type II hydropic (both patients) \\
\hline Head/facial features & $\begin{array}{l}\text { Coarse facies } \\
\text { Deafness }\end{array}$ & $\begin{array}{l}\text { Facial dysmorphism } \\
\text { Low-set ears (bilateral hearing loss) } \\
\text { Brachycephaly }\end{array}$ & $\begin{array}{l}\text { Coarse facies* } \\
\text { Bushy eyebrows* } \\
\text { Depressed nasal bridge* } \\
\text { Wide nose* }\end{array}$ \\
\hline Eyes & Orbital hypoplasia & Not reported & Corneal opacity* \\
\hline Mottled skin & Not reported & Yes & Yes* \\
\hline Organomegaly & $\begin{array}{l}\text { Yes } \\
\text { (hepatosplenomegaly) }\end{array}$ & $\begin{array}{l}\text { Yes (hepatosplenomegaly, and } \\
\text { glomerular epithelial were enlarged) }\end{array}$ & Yes (hepatosplenomegaly)* \\
\hline Ascites/edema & Yes (abdomen) & $\begin{array}{l}\text { Yes (abdomen and glomerular epithelial } \\
\text { contained large amounts of foamy) }\end{array}$ & $\begin{array}{l}\text { Yes (abdomen in both patients and bilateral } \\
\text { hydrocele)* }\end{array}$ \\
\hline Muscle & Not reported & Not reported & Hypotonia* \\
\hline Pneumopathy & Not reported & Yes (right upper lobe pneumonia) & Yes (both patients) \\
\hline Heart & Not reported & Yes (mildly enlarged) & Yes (ventricular septal hypertrophy, aortic dilation)* \\
\hline Nervous system & Yes (DD) & $\begin{array}{l}\text { Yes (DD, bilateral mild frontotemporal } \\
\text { atrophy) }\end{array}$ & $\begin{array}{l}\text { Yes* (DD, most likely in Patient } 2 \text { too); corpus } \\
\text { callosum hypoplasia*; cerebral atrophy (patient 2) }\end{array}$ \\
\hline Skeleton & Craniosynostosis & Bilateral hip dysplasia & $\begin{array}{l}\text { Spinal bone dysostosis* } \\
\text { Column with dorsal hump* } \\
\text { Wide sacral fossa* }\end{array}$ \\
\hline Hernia & Not reported & No & Bilateral inguinal hernia (both patients) \\
\hline
\end{tabular}

* Clinical features confirmed only in Patient 1. DD, developmental delay.

hitherto been reported. Although most of the features observed in our patients are part of the clinical spectrum of sialidosis type II, and some of these overlap with the clinical features (including reduced survival) of the previously reported patients with the same pathogenic variant, the corpus callosum hypoplasia, corneal opacity, hernia, dysostosis and ventricular septal hypertrophy had not been reported in them (Table 1) [Bonten et al., 2000; Maroofian et al., 2018]. In the absence of further pathogenic or likely pathogenic variants (at least those affecting coding or splice regions in the index case's exome) within NEU1, CTSA, or GLB1 that could directly modify the impact of Y370C, these results suggest incomplete penetrance and/ or variable expressivity.

Clinical Exome Sequencing in Congenital

Sialidosis Type II Diagnosis
According to our molecular modeling results, the tridimensional structure of the substrate-binding pocket of sialidase- 1 , which contains 5 highly conserved amino acids among all neuroaminidases analyzed so far (R76, R278, R339, E392, and E262 in our model), is not drastically modified by the Y370C substitution (Fig. 2a-d). However, the highly conserved amino acid Y370 is essential for the stabilization of a carbonium ion intermediate of the sugar ring of the substrate during catalysis. In contrast, the pathogenic variant $\mathrm{C} 370$ appears to be unable to reach sufficiently into the pocket and therefore cannot properly stabilize the intermediate state, eliminating the catalytic activity of NEU1 as a result. Although the resulting sialidase- 1 appears to have a decreased stability [Bonten et al., 2000] and appears to be able to reach into the 
lysosome compartment, currently it is unknown whether this change affects multiprotein complex formation. The fact that parents of the patients along with the other individual of Latino ancestry are heterozygous for the sequence variant and asymptomatic (assumed for the latter), suggests that sialidase- 1 is haplosufficient and forms functional protein complexes without negative dominant effects from the mutant one.

In conclusion, our genomic study along with the clinical presentation, allowed us to diagnose both patients with the congenital/hydropic subtype of sialidosis type II. In addition, we present the atomic modeling of the mutant protein to increase our understanding of the ultra-rare pathogenic variant Y370C. Thus, overall, our findings provide additional information that may serve to diagnose other similar patients carrying such a variant. Moreover, given the complex clinical picture of sialidosis patients and the overlap of its clinical features with other disorders, as well as the limitation of routine tests in our country, this work further supports the prompt use of high-throughput platforms to reach a timely diagnosis. This supports genetic counseling in health systems from middle- and low-income countries. In line with this, WES is more cost-effective compared to biochemical tests, since the processing time for the former is faster, it is relatively cheaper, and it generates much more information in only one assay. Finally, this effort would lead to early access to treatments to improve the quality of life of patients affected by these (and other) rare disorders.

\section{Acknowledgements}

We thank the parents who continuously supported this research. E.A.F.-C. was supported by a CONACyT [scholarship No. 334775]. The authors also wish to thank Jair S. García-Sotelo, Alejandro de León, Carlos S. Flores, and Luis A. Aguilar of the Laboratorio Nacional de Visualización Científica Avanzada from the National Autonomous University of Mexico, and Alejandra Cas- tillo, Carina Díaz, Abigayl Hernández and Eglee Lomelin of the International Laboratory for Human Genome Research, UNAM. C.C.-G. is a PhD student from Programa de Doctorado en Ciencias Biomédicas, Universidad Nacional Autónoma de México (UNAM) and is supported by CONACyT [scholarship No. 385365].

\section{Statement of Ethics}

The patients' parents were informed about genetic studies and signed the respective informed consent of their children and themselves. This study is included in a research protocol, which was approved by a local ethical committee of the School of Medicine of Universidad Autonoma de Nuevo Leon [BI15-003], and it is in accordance with the ethical principles of medical research of the Declaration of Helsinki (1964) and its further amendments.

\section{Conflict of Interest Statement}

The authors have no conflicts of interest to declare.

\section{Funding Sources}

This work was supported by CONACYT (INFRA-2013-204423 and S0008-2014-1-233212) for C.C.-F.

\section{Author Contributions}

C.C.-F. conceived the study, proposed the experiments, critically analyzed and interpreted the results of WES, and wrote part of the manuscript. E.A.F.-C. performed WES experiments and wrote part of the manuscript. V.Z.-S. and R.V. carried out the in silico protein model and contributed the theoretical part. J.E.G.O., A.A.H.-O., L.E.B.-S., and E.E.-G. participated in the diagnosis, description, biochemical tests, amniotic karyotyping, and discussion of the clinical features, as well as provided patients' samples. C.D.R.-E., C.C.-G., and C.M.-A. contributed with the design and experiments of Sanger sequencing, as well as critically revised the manuscript. All authors read and approved the final version of the manuscript.

\section{References}

Annunziata I, d'Azzo A. Galactosialidosis: historic aspects and overview of investigated and emerging treatment options. Expert Opin Orphan Drugs. 2017;5(2):131-41.

Arora V, Setia N, Dalal A, Vanaja MC, Gupta D, Razdan T, et al. Sialidosis type II: Expansion of phenotypic spectrum and identification of a common mutation in seven patients. Mol Genet Metab Rep. 2020;22:100561.
Artimo P, Jonnalagedda M, Arnold K, Baratin D, Csardi G, de Castro E, et al. ExPASy: SIB bioinformatics resource portal. Nucleic Acids Res. 2012;40(Web Server issue):W597-603.

Bonten E, van der Spoel A, Fornerod M, Grosveld G, d'Azzo A. Characterization of human lysosomal neuraminidase defines the molecular basis of the metabolic storage disorder sialidosis. Genes Dev. 1996;10(24):3156-69.
Bonten EJ, Arts WF, Beck M, Covanis A, Donati MA, Parini R, et al. Novel mutations in lysosomal neuraminidase identify functional domains and determine clinical severity in sialidosis. Hum Mol Genet. 2000;9(18):2715-25.

Crennell SJ, Garman EF, Laver WG, Vimr ER, Taylor GL. Crystal structure of a bacterial sialidase (from salmonella typhimurium LT2) shows the same fold as an influenza virus neuraminidase. Proc Natl Acad Sci USA. 1993;90(21):9852-6. 
d'Azzo A, Machado E, Annunziata I. Pathogenesis, Emerging therapeutic targets and Treatment in Sialidosis. Expert Opin Orphan Drugs. 2015;3(5):491-504.

Franceschetti S, Canafoglia L. Sialidoses. Epileptic Disord. 2016;18(S2):89-93.

Guex N, Peitsch MC, Schwede T. Automated comparative protein structure modeling with SWISS-MODEL and Swiss-PdbViewer: A historical perspective. Electrophoresis. 2009; 30 Suppl 1:S162-73.
Khan A, Sergi C. Sialidosis: A Review of Morphology and Molecular Biology of a Rare Pediatric Disorder. Diagnostics. 2018;8(2):29.

Maroofian R, Schuele I, Najafi M, Bakey Z, Rad A, Antony D, et al. Parental Whole-Exome Sequencing Enables Sialidosis Type II Diagnosis due to an NEU1 Missense Mutation as an Underlying Cause of Nephrotic Syndrome in the Child. Kidney Int Rep. 2018;3(6):145463.
Pshezhetsky AV, Richard C, Michaud L, Igdoura S, Wang S, Elsliger MA, et al. Cloning, expression and chromosomal mapping of human lysosomal sialidase and characterization of mutations in sialidosis. Nat Genet. 1997; 15(3):316-20.

Yogalingam G, Bonten EJ, van de Vlekkert D, Hu $\mathrm{H}$, Moshiach S, Connell SA, et al. Neuraminidase 1 is a negative regulator of lysosomal exocytosis. Dev Cell. 2008;15(1):74-86. 